\title{
THE ACTION OF CHLORINE, ETC., ON THE BRONCHI REPORT TO THE CHEMICAL WARFARE COMMITTEE
}

\author{
BY J. A. GUNN \\ (From the Department of Pharmacology, University Museum, Oxford)
}

With Plates 2 and 3

THE Chemical Warfare Committee suggested to me the following subjects for investigation: (1) the effect of chlorine inbalation on the calibre of the bronchi and (2) the utility of drugs that could be used to counteract whatever effect chlorine was found to produce. I have not thought it necessary in this report to deal with the literature of the subject or to do more than give a summary of my observations. It is not a matter of great difficulty to determine the action on the bronchi of drugs that can be administered intravenously, especially if they act peripherally; and various methods devised for this purpose are in ordinary use. The difficulties multiply when one begins to apply those methods to the investigation of a gas like chlorine, and some of those difficulties are mentioned in the report. Such methods as I was able to devise for obtaining some indication of the effects of known concentrations of the gas are not entirely free from objection, but I have described in some detail those I found most reliable because it is possible that, for investigating the action of gases that do not corrode metal or rubber, they could be applied with such slight modification as would remove the objections mentioned.

\section{A. Methods of recording Changes in the Calibre of the Bronchi.}

Of the various methods that have been employed for recording changes in the calibre of the bronchi, none are very direct, and most of them depend upon the fact that, with constant force used to expand the lungs-e.g. by positive artificial respiration through the trachea-the amount of excursion of the lungs varies with the sectional area of the lumen of the bronchioles. The amplitude of the lung movement can be graphically recorded. I have used chiefly the following methods. The experiments were made on anaesthetized rabbits or decerebrated cats. Artificial respiration was carried out by Brodie's pump, which delivers a sufficiently regular blast of air, that could be warmed or charged with a volatile anaesthetic as required.

[Q. J. M., Jan., rg20. ] 
The following device for recording the expansion of the lungs was found after many experiments to be most successful (see Diagram I). A circular opening was made in the chest just above the diaphragm. The opening was about half an inch in diameter, and haemorrhage was prevented by ligatures round the ribs and sternum where required. An inverted thistle-funnel was tied into this opening. The exit tube of the thistle-funnel was connected with a tambour, c, with a T-tube, $\mathrm{B}$, intervening. The tambour was covered with a slack membrane (thin rubber loosely tied). The T-tube had a clip, D, on the tubing attached to the vertical limb, by means of which the excursion could be regulated to a convenient size. By this method one really measures the 'jerk' imparted to the tambour by the expanding lung. The actual volume of the lung is not

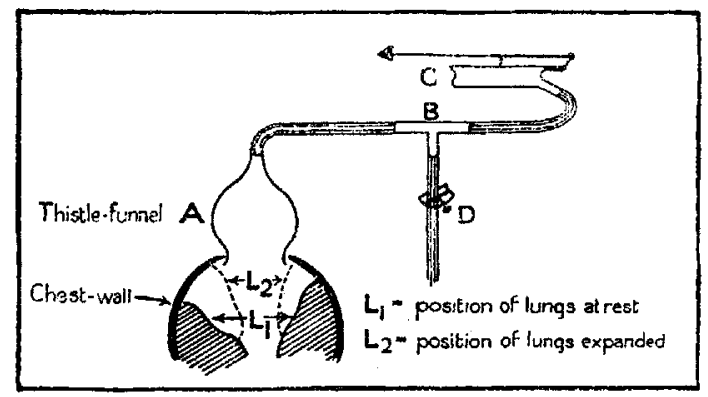

Dragram I.

recorded, as the system is not closed; having an escape at, $D$, one avoids any excessive positive pressure on the outer surface of the lung. With constant artificial respiration, the excursion of the tambour is constant. If the bronchioles dilate the lung expands farther and faster and the ' jerk' is increased; contrariwise, when the bronchioles contract. Fig. 1, Plate 2, illustrates the record one obtains, showing the regularity of the normal excursion and the marked reduction produced by intravenous injections of a minute dose of ergamine. This use of a thistle-funnel instead of a cannula in the pleural cavity is important. I have tried the various kinds of cannulae in use and none of them are reliable. They may give misleading tracings by the mouth of the cannula being blocked by the expanding lung. This cannot happen with a thistle-funnel unless one uses an excessive expansion of the lung. The transparency of the funnel also allows one to see to some extent what is actually happening to the lung.

\section{B. Method of introducing a Known Concentration of Gas into the Air respired.}

The problem was how to introduce into the lungs at will a known concentration of gas, while keeping constant the blast that entered the tracheal cannula. One of the chief difficulties is that one cannot pass chlorine through a metal cylinder without injuring the cylinder, nor can one pass it through anything but a small length of rubber tubing, as the latter takes up the chlorine. 
Diagram II illustrates the method adopted. The air pumped by the Brodie's pump was made to pass through a carboy before entering the trachea. By means of the connexions shown, while the normal tracing was being taken the air passed along the route $A, B, C, E$. When the action of the gas was to be tested the rubber tube $\mathrm{C}$ was closed with a clip and, by opening two other clips, the air passed through the other limbs of the $Y$-tubes $B$ and $\mathrm{E}$, between which was a glass tube $\mathrm{D}$ full of pure chlorine. This chlorine was then driven into the carboy and so into the lungs. By keeping the width of the glass and rubber tubing the same throughout, it was found by experiment (the tube $\mathrm{D}$ in this case containing air only) that no change in the lung excursion was mechanically produced by changing from $c$ to $\mathrm{D}$. The carboy had a volume of 45 litres, and different

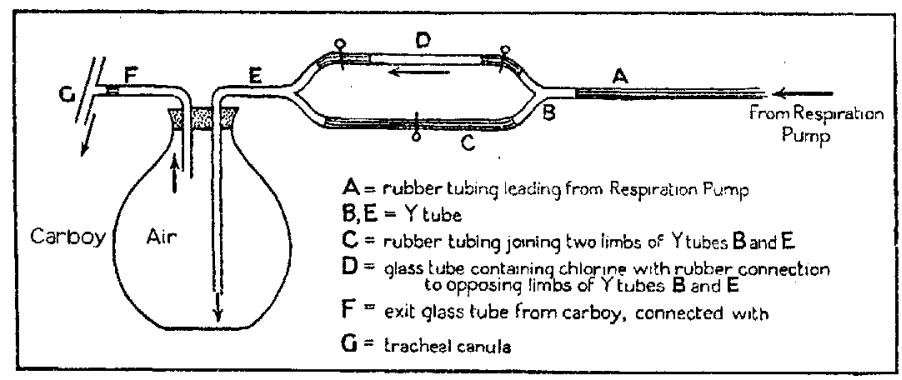

DiagRAM II.

concentrations of chlorine were obtained by cutting different lengths of tube $\mathrm{D}$, the capacity of which could of course be easily measured. In the apparatus as set up, the chlorine passed through glass with the exception of about $1 \frac{1}{2}$ inches of rubber tubing. The rhythmic pumping served to mix the chlorine in the carboy before it reached the trachea. The concentration of chlorine rapidly fell as more air was pumped through the carboy, but it is difficult to devise for such experiments any method by which this can be avoided. The chief drawback of this method is that, when the air is pumped through a large carboy on its way to the trachea, this reservoir tends to convert an intermittent into a constant blast of air. It was possible to prevent this to a sufficient degree to get some satisfactory experiments, but in many cases, owing to insufficient ventilation of the lungs, natural respiration began in spite of artificial respiration, in which case the tracing was too irregular to be satisfactory. However, by controlling the results with the following method, one could obtain a sufficiently certain indication of the effects of chlorine on the bronchi.

\section{Contral Method.}

The results of experiments obtained by the above method were compared with those obtained in the following way (see Diagram III).

The animal breathedinaturally. The tracheal cannula was provided with an expiratory valve which allowed air to be breathed out but not inspired. The air 
was inspired through a carboy as figured. A normal tracing was first taken, and then by means of a glass syringe a known volume of chlorine was injected into the carboy and the animal then breathed air containing a known concentration of chlorine instead of pure air. The effect on the respiration was determined by a respiratory stethograph, and the effect on the bronchi could to some extent be estimated by comparing the stethograph record with a record which was simultaneously taken of the intrapleural pressure.

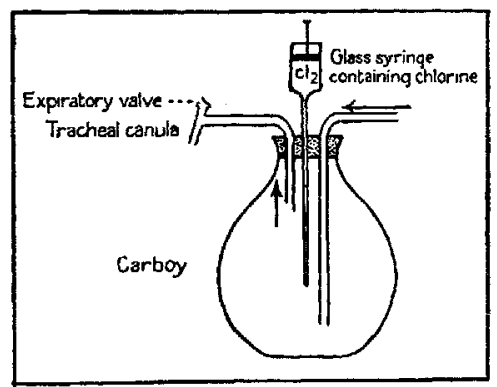

Diagram III.

\section{Action of Chlorine on the Bronchi.}

It was theoretically probable that any effect exerted by chlorine on the musculature of the bronchi would be a reflex one, and in any case the possibility of this occurring had to be allowed for. It was necessary, therefore, that the excitability of the respiratory centre should. be maintained in a condition as normal as possible. In all the experiments on cats, the animals were decerebrated by Sherrington's method. After decerebration normal respiration is resumed, and the effect of chlorine was not tried until at least half an hour after decerebration, by which time there would be eliminated most of the anaesthetic (chloroform) which had been used before decerebration. Shortly after decerebration, artificial respiration was begun, and after a short time normal respiration ceased, but would immediately begin if artificial respiration were interrupted. The animals were therefore in good condition as regards the activity of the respiratory centre and it was reasonable to assume that reflex effects on the bronchi were elicitable. In rabbits urethane was tried as an anaesthetic. Brodie and Dixon have stated that it interferes with bronchial reactions, preventing the exhibition of constrictor and dilator effects by drugs which show these effects in the absence of urethane. This I also found, and abandoned urethane as an anaesthetic. In rabbits I therefore used chloroform-ether until the operation was over, and then ether alone. Fig. 2, Plate 3, shows the absence of effect of ergamine on the bronchi of a rabbit anaesthetized by urethane (ef. Fig. 1). After experiments with many drugs I found ergamine the most certain in its action on the bronchi, and I therefore employed it in testing various methods, \&c. I found that chlorine in concentrations of 1 in 5,000 to 1 in 1,000 produces a short-lasting reflex constriction of the bronchi. This occurs immediately it is inhaled, and does not last more than 
a minute or so. Fig. 3 shows the effect of chlorine, 1 in 2,000, on the bronchi and blood-pressure of a decerebrated cat. The effects on the bronchi were determined by artificial respiration and the thistle-funnel method of recording. There was a brief broncho-constriction, lasting about one minute, accompanied by a fall of blood-pressure.

Fig. 4 shows the effect of the same concentration of chlorine on a rabbit, breathing naturally. A record was taken of the respiratory movements by stethograph, and of the intrapleural pressure and of the blood-pressure. The inhalation of chlorine produced a diminution of the amplitude of the movements, the inspiratory movement being increased and the expiratory diminished. But coincident with this the positive as well as the negative pressure in the thorax was increased. This is typical of what happens when the bronchi are constricted (e.g. in spasmodic asthma), the chest tending to become fixed in the inspiratory position. Owing to the constriction of the bronchi, the less vigorous expiratory effort is unable to empty the lungs and the excursion of the chest in expiration is lessened. But the actual expiratory effort is usually increased, so that though the expiratory movement is diminished, the positive intrathoracic pressure is increased by expiration. There can be little doubt that the tracing therefore indicates a short-lasting bronchial constriction. Tho respiration is simultaneously quickened. The acceleration of respiration lasts for a much longer period than the bronchial constriction As has been noted by other observers, the blood-pressure falls.

Generally, the results of experiments with chlorine have gone to show that inhalation of 1 in 5,000 up to 1 in 1,000 produces an increased rate of respiration with a transient broncho-constriction. This broncho-constriction is produced reflexly by the first contact of the irritant vapour with the bronchial mucous membrane. It lasts such a short time that therapeutic measures to combat it would be unavailing. A subsequent sudden increase in the concentration of the gas may produce another transient reflex broncho-constriction. Continued inhalation of the gas produces thereafter an apparent slight and gradual bronchoconstriction, but from histological observations it appears probable that this is due rather to oedema of the bronchial mucous membrane than to contraction of the bronchial muscle.

It is perhaps well to keep' in mind that reflex effects arising from irritation of the upper air-passages are excluded in these experiments. It is theoretically probable that irritation of the nasal and laryngeal mucous membranes will produce more immediate and possibly more intense reflex effects on the bronchi than will irritation of the trachea; for the sentinel posted in the latter region challenges the enemy gas too late.

\section{The Action of some Drugs on the Bronchi.}

In the course of these experiments, while various methods were being tried, a number of observations were made on the action of drugs. Of these observations perhaps the following bave some bearing on the present line of investigation and 
may be of some assistance to others engaged on it. Of the drugs I tried-and I tried most of those which are reputed to possess this action-the most certain broncho-constrictor is ergamine, the action of which has been fully described by Dale. It has this advantage, that it produces a fall of blood-pressure in the cat and a rise in the rabbit, so that the fact that the bronchial changes recorded are independent of vascular changes in the lung can be arrived at without other elaborate proof. If the dose employed be small, e.g. about 0.1 or $0.2 . \mathrm{mg}$. per kilo, the effect can be repeated several times. If the dose be much above that, the bronchi remain constricted during the usual time of an experiment.

Morphine. The broncho-constrictor action of morphine and its derivatives has been emphasized especially by Jackson. His experiments were performed chiefly on dogs. In experiments on rabbits and cats, I have found that doses corresponding to ten times the therapeutic dose per kilogram used in man produce only a slight broncho-constriction. Unless the quantitative reaction in man differs widely from that in those animals, the effect of therapeutic doses of morphine on the bronchioles is too slight to contra-indicate the use of morphine (in gas-poisoning for example), should it be found for other reasons necessary to employ it.

Broncho-dilators. Though, in the acute stage of chlorine poisoning, broncho dilators seemed to be of no practical utility, it was thought likely that other poisonous gases might produce more distinct and lasting broncho-constriction, for the relief of which broncho-dilators would be valuable. Of the three most commonly used broncho-dilators-atropin, adrenalin, and nitrites-atropin in sufficient doses seemèd to be most efficacious in removing a bronchoconstriction produced reflexly or by stimulation of the vagus nerve in any part of its course. If the bronchial secretion is considerably increased coincidently with the bronchial constriction, as for example by pilocarpine, atropin usually does not completely remove the obstruction to the entry of air into the lung. This is probably due not to its failure to dilate the bronchi but to its diminishing the bronchial secretion, rendering it more viscid and thus blocking the air-way. On the other hand, adrenalin, which dilates the bronchi by stimulating the sympathetic nerve-ends instead of, like atropin, by paralysing the vagus terminals, has no marked effect on the bronchial secretion, and is thus safer for general use. One point in regard to the action of adrenalin on the bronchi may be mentioned. It is generally stated that adrenalin dilates the bronchi only when they are previously constricted by, e.g., pilocarpine. This statement is too absolute. In the decerebrate cat, if sufficient time is allowed for elimination of the anaesthetic, adrenalin produces a slight but definite dilation of the bronchi, even in the absence of any artificial constriction. This result is more credible because otherwise it would mean that the bronchi are normally in a condition of maximum dilation, and, if so, lose the benefit of their double nerve supply under ordinary conditions. The mechanism would certainly be more flexible if the calibre of the bronchi was normally an intermediate one, capable of either increase or diminution as the result of sympathetic or vagus activity respectively. 
This apparently is not the case, though, so far as can be judged from such experiments, the normal calibre seems to be somewhere near that of maximum dilatation. In my experiments nitrites (inhalation of amyl nitrite or intravenous injection of sodium nitrite) produced a relatively slight dilatation of the bronchi even in doses which caused a profound fall of blood-pressure. For practical purposes they appear to be much less valuable than either atropin or adrenalin.

Urethane. Considering the remarkable effect that uretbane, when used as a general anaesthetic, exerts in preventing the occurrence of broncho-constriction which would normally be produced by ergamine, arecolin, \&c., it was thought possible that intravenous injection of smaller doses of urethane might be able to dilate the bronchi when previously constricted by those drugs, in which case it might have proved of clinical value as a broncho-dilator. Experimentally this was not found to be the case; at least it certainly has no immediate effect in dilating the bronchi previously constricted. When urethane is used as an anaesthetic it is usually allowed at least half an hour to develop its anaesthetic effects before the animal is used for experiment. The probability is that its action on the bronchi-which is not yet sufficiently explained-takes a considerable time to develop. It does not appear, therefore, to possess any practical value for producing an immediate dilatation of the bronchi.

\section{DESCRIPTION OF TRACINGS.}

Plate 2, Fia. 1. Rabbit under chloroform-ether anaesthesia. Funnel-tambour record of lung expansion with artificial respiration. Showing diminution of lung expansion (constriction of bronchi) by ergamine.

Plate 3, Fra. 2. Rabbit under deep urethane anaesthesia. Conditions of experiment otherwise the same as in Fig. 1. Showing absence of effect of ergamine on bronchi.

Fia. 3. Cat decerebrate. Funnel-tambour record of lung expansion with artificial respiration. Showing transient reflex constriction of bronchi by inhalation of chlorine, 1 in 2,000 .

FIG. 4. Rabbit under ether anacsthesia; natural respiration.

R. $=$ Stethograph record of respiration, upstroke = inspiration.

I.P. $=$ intrapleural pressure, downstrcke $=$ negative pressure $=$ inspiration.

B.P. $=$ blood-pressure.

Showing constriction of bronchi, acceleration of respiration, and fall of blood-pressure by inhalation of chlorine, 1 in 2,000 . 


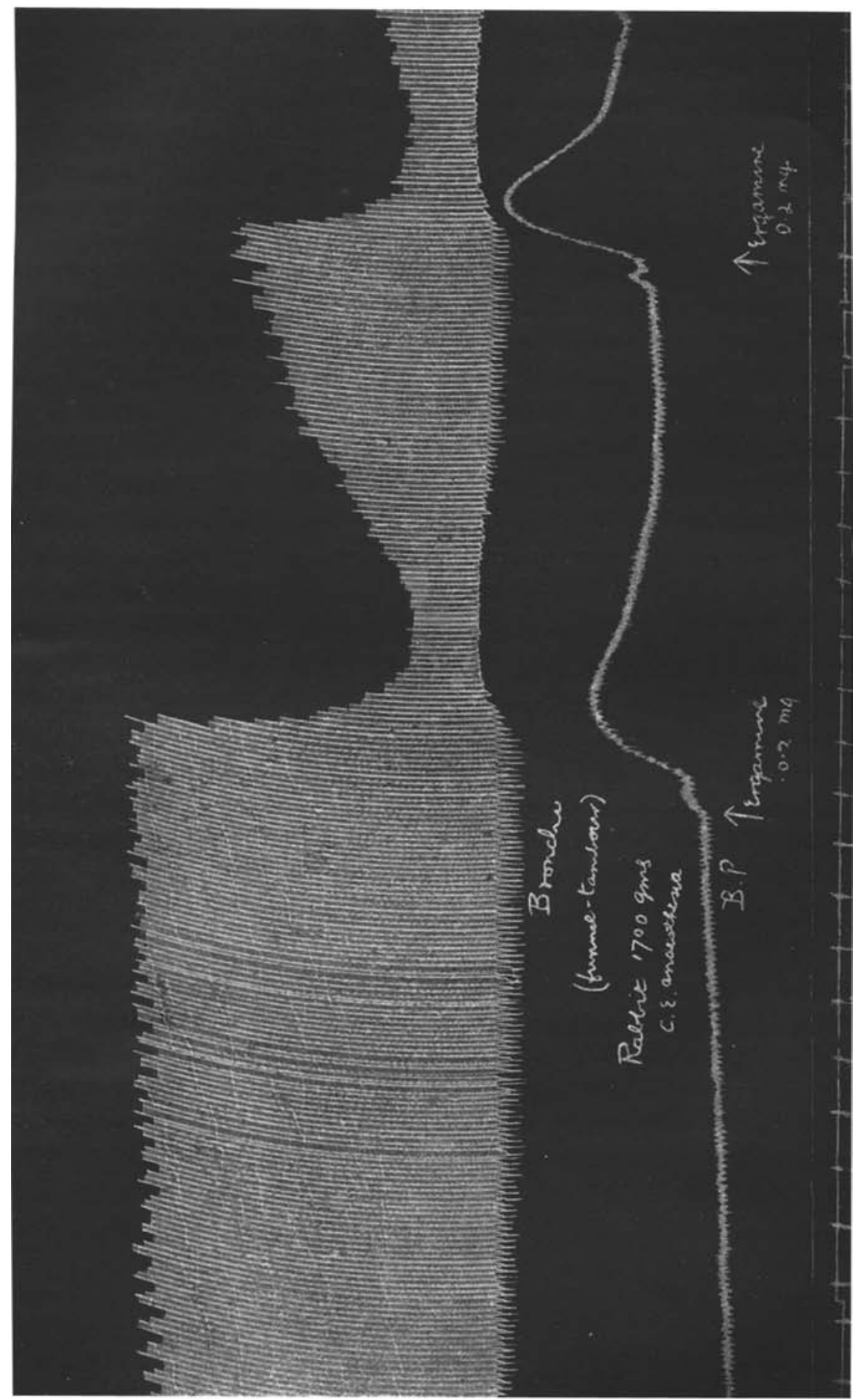

$\stackrel{-}{\circ}$ 


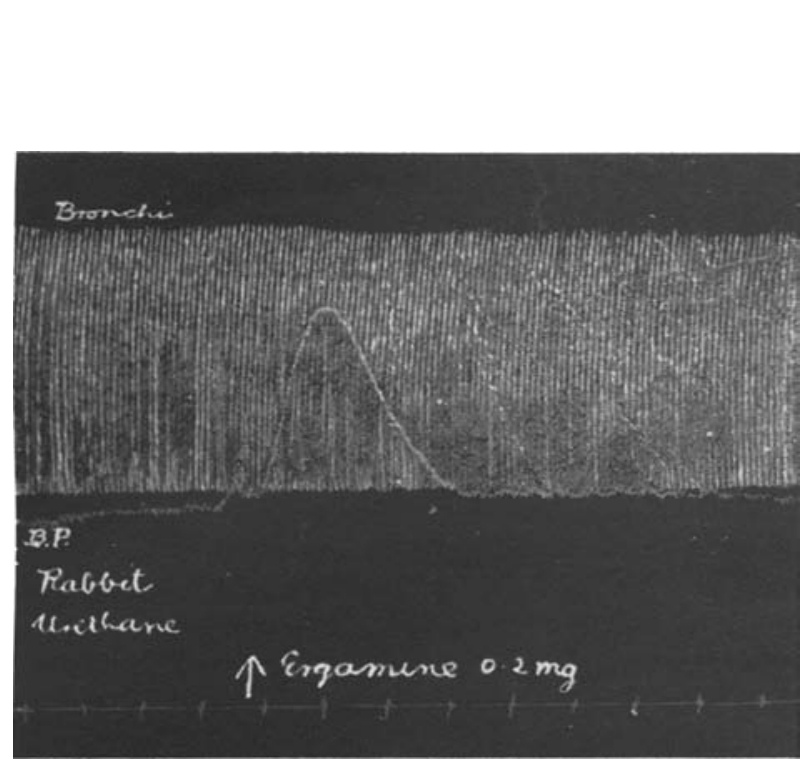

FIG. 2

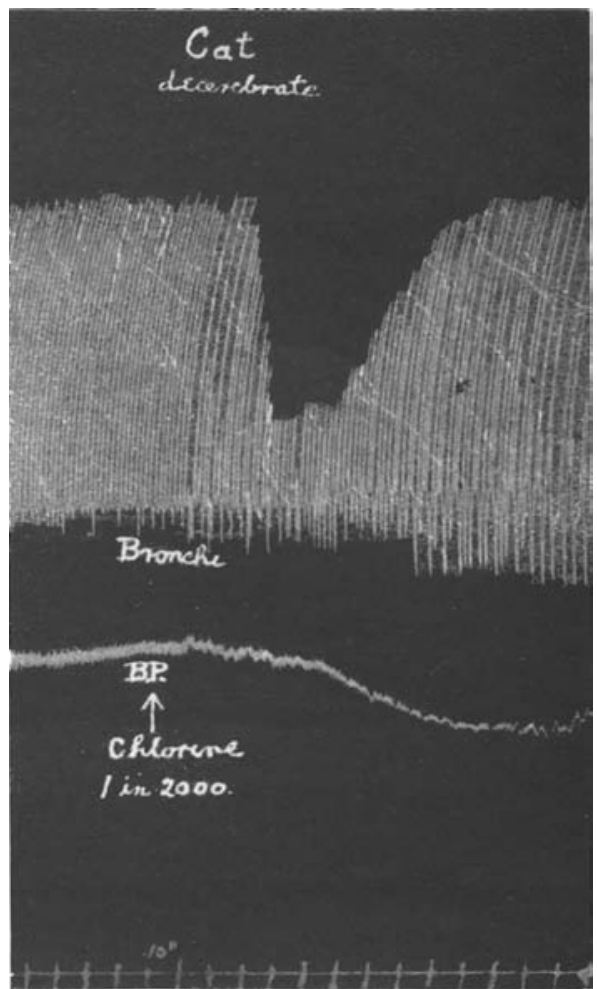

FIG. 3.

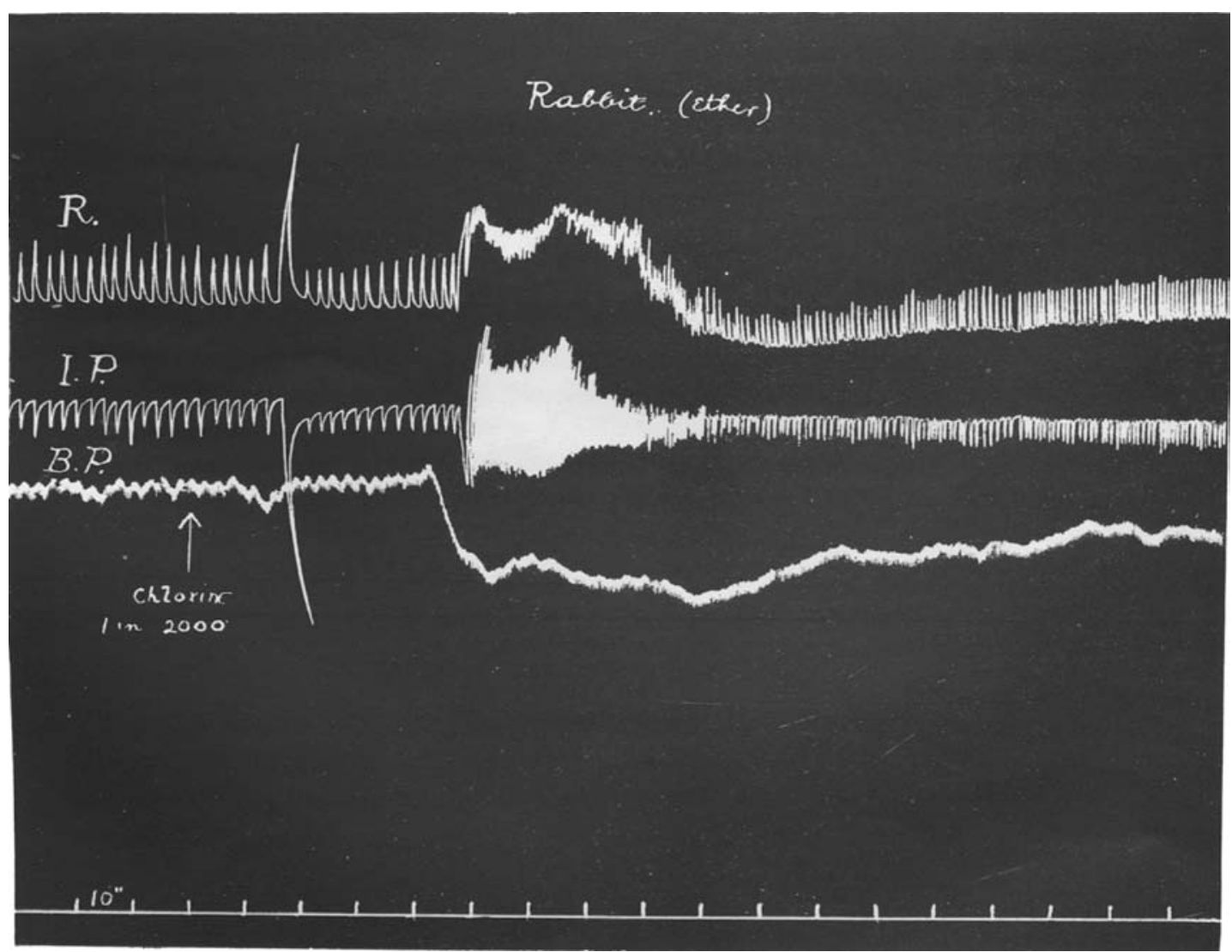

FIG. 4. 